\title{
Serum Apolipoprotein B/apolipoprotein A1 Ratio in Relation to Intervertebral Disc Herniation : A Cross- sectional Frequency-Matched Case-Control Study
}

\section{Fei Chen}

Department of Cardiovascular, Pingxiang people's hospital, Pingxiang 337000, Jiangxi Province, P. R. China.

\section{Tongde Wu}

Department of Spinal Surgery, Shanghai East Hospital, Tongji University School of Medicine, 150 JiMo Road, Shanghai 200120, China.

\section{Song Guo}

Department of Orthopedics, Shanghai General Hospital, Shanghai Jiao Tong University School of Medicine, Shanghai 200080, P. R. China.

\section{Junwen Huang}

Department of Cardiovascular, Pingxiang people's hospital, Pingxiang 337000, Jiangxi Province, P. R. China.

\section{Qiang Fu}

Department of Orthopedics, Shanghai General Hospital, Shanghai Jiao Tong University School of Medicine, Shanghai 200080, P. R. China.

\section{Yaqing pan}

Department of Spinal Surgery, Shanghai East Hospital, Tongji University School of Medicine, 150 JiMo Road, Shanghai 200120, China.

\section{Huiying Zhang}

Department of Spinal Surgery, Shanghai East Hospital, Tongji University School of Medicine, 150 JiMo Road, Shanghai 200120, China.

\section{Desheng Wu}

Department of Spinal Surgery, Shanghai East Hospital, Tongji University School of Medicine, 150 JiMo Road, Shanghai 200120, China.

\section{Qi Chen}

Department of Cardiovascular, the Second Affiliated Hospital of Nanchang University, Nanchang 330000, Jiangxi Province, P. R. China.

\section{lijun Li}

Department of Spinal Surgery, Shanghai East Hospital, Tongji University School of Medicine, 150 JiMo Road, Shanghai 200120, China.

Xinhua Li ( $D$ xinhuali1013@163.com ) 
Shanghai General Hospital Department of Orthopaedics: Shanghai Jiaotong University First People's Hospital Department of Orthopaedics

\section{Research}

Keywords: Intervertebral disc herniation, serum lipid, dyslipidemia, Lp(a), Apo B/Apo Al

Posted Date: November 25th, 2020

DOl: https://doi.org/10.21203/rs.3.rs-113468/v1

License: (c) (1) This work is licensed under a Creative Commons Attribution 4.0 International License. Read Full License

Version of Record: A version of this preprint was published at Lipids in Health and Disease on July 29th, 2021. See the published version at https://doi.org/10.1186/s12944-021-01502-z. 


\section{Abstract}

\section{Background}

In recent decades, the serum lipid profile of apolipoprotein(a) $(L p(a))$ level and apolipoprotein $\mathrm{B} /$ apolipoprotein $\mathrm{A} 1$ ratio (Apo B/ApoA1) ratio were found more representative for serum lipid level and were recognized as the independent risk factors for various diseases. Although the serum lipid levels of total cholesterol (TC), triglycerides (TG), low-density lipoprotein cholesterol (LDL-C), high-density lipoprotein cholesterol (HDL-C) were found associated with symptomatic IDH, no studies have been conducted to date for the evaluation of the association of $A p o A l, A p o B, L p(a)$ and $A p o B / A p o$ Al levels with symptomatic IDH.

Materials

A total of 1,839 Chinese patients were recruited in the present study. 918 patients were diagnosed as IDH cases and were enrolled in the experimental group. A control group of 921 patients underwent a physical examination during the same period. The serum lipid levels of TC, TG, LDL-C, HDL-C, Lp(a), Apo B and Apo B/Apo Al were examined and analyzed.

Results

The patients in the control group were collected randomly from patients who were matched with the baseline levels of the aforementioned lipid molecular. The patients with IDH exhibited significantly higher TC, TG, LDL, Apo B and Lp(a) levels compared with the control subjects. The percentage of high-TC, highTG, high-LDL, high-Apo B and high-Lp(a) were significantly higher in the IDH group. However, hyperlipidaemia was not associated with the degenerated segment of the IDH ( $P=0.201)$. The odds ratios (OR) for the incidence of IDH with an elevated LDL-C, TC, TG, Lp(a), Apo B and Apo B/Apo Al were 1.583, $1.74,1.62,1.58,1.49$ and 1.39 , respectively. The correlation analysis revealed the correlation between elevated $L D L-C, T C, T G, A p o B, L p(a)$ and incidence of IDH was significant $\left(R^{2}{ }_{L D}=0.017 ; R^{2} T C=0.004\right.$; $\left.\mathrm{R}^{2}{ }_{\mathrm{TG}}=0.015 ; \mathrm{R}^{2}{ }_{\text {apo }}=0.004 ; \mathrm{R}_{\mathrm{LP}(\mathrm{a})}=0.021\right)(\mathrm{P}<0.05)$.

\section{Conclusions}

The present study suggests that elevated levels of serum TC, TG, LDL, Apo B, Lp(a) and Apo B/Apo Al are associated with a higher risk for IDH.

\section{Background}

Intervertebral disc degeneration (IVDD) and related diseases are a leading cause of disability, notably jobrelated disability[1-3]. Despite the extensive investigation of the etiology and treatment of IVDD, the underlying pathophysiological mechanisms remain unclear[3-8]. A variety of risk factors, such as excessive mechanical overload, aging, tissue injury, smoking, abnormal metabolism levels, and genetic 
risk have been found to contribute to the onset and progression[9-11]. Among these factors, abnormal lipid metabolism and atherosclerosis (AS) have been implicated in the development of symptomatic intervertebral disc herniation (IDH) and/or low back pain[12-16]. A Finnish study performed by Leino-Arjas al[12] identified that the positive correlation between higher total cholesterol (TC), low density lipo-protein cholesterol (LDL-C), triglycerides (TG) levels and sciatica. Several studies further demonstrated that triglycerides (TG) and total cholesterol (TC) were associated with the severity of low back pain and symptomatic IDH[13-18].

With the increased research on serum lipid, the liporoprotein fraction of apolipoproteinAI (Apo Al) and apolipoprotein B (Apo B) and the ratios of apolipoprotein B/apolipoprotein Al (Apo B/Apo Al), and apolipoprotein(a) $(\mathrm{Lp}(\mathrm{a}))$ have received considerable attention in the investigation of dyslipidaemia related diseases in recent years, and $A p o B / A p o ~ A l$ and $L p(a)$ were recognized as the independent risk factors for various diseases, including osteoarthritis and AS[19-21] . However, the possible association between Apo Al, Apo B, Lp(a) and symptomatic IDH remains undiscovered.

In this study, a frequency-matched case-control study of serum lipid levels obtained from patients with symptomatic IDH was conducted in order to evaluate the relationship between serum lipid levels and symptomatic IDH in our study. We found that the patients with IDH exhibited significantly higher TC, TG, LDL, Apo B and Lp(a) levels compared with the control subjects. The percentage of high-TC, high-TG, high-LDL, high-Apo $B$ and high-Lp(a) were significantly higher in the IDH group. Moreover, the odds ratios (OR) analysis revealed elevated LDL-C, TC, TG, Lp(a), Apo B and Apo B/Apo Al had a higher risk of IDH incidence. The correlative analysis further suggested elevated LDL-C, TC, TG, Lp(a), Apo B and Apo B/Apo Al are significantly closely with incidence of IDH. Our findings revealed that elevated levels of serum TC, TG, LDL, Apo B, Lp(a) and Apo B/Apo Al are associated with a higher risk for IDH.

\section{Methods}

All procedures described in the present study were approved by the Ethics Committee of our institution. All patients provided written informed consent. The detailed primary flow-charts were presented in Figure 1. A total of 4,349 patients accepted the MRI scan and were potentially considered for inclusion from 2010 to 2019 in our institution. A total of 3,431 patients were excluded due to failure to meet the inclusion criteria (history of spinal disorders ( $n=155)$, multiple IDHs $(n=367)$, spondylolysis $(n=98)$, foraminal or central canal stenosis $(n=187)$, spinal trauma $(n=67)$, primary osteoarthritis of the operated and/or contralateral joint $(n=367)$, inflammatory joint disease $(n=211)$, diabetes $(n=311)$, coronary heart disease $(n=516)$, cerebrovascular disease $(n=217)$, hypertension $(n=467)$, smoking $(n=468)$ ). A total of 918 patients (399 men and 519 women; mean age: $60.74 \pm 12.69$ years, range 18 to 93years) met our inclusion criteria and were enrolled in our study in group 1 (symptomatic IDH group). A total of 921 patients (control group) (401 men and 520 women; mean age: 61.02 12.59 years, range 18-91years), who underwent physical examination and conducted the MRI scan during the same period, were matched with the baseline of the symptomatic IDH group (Table 1). The patients in the control group were excluded for IVDD by the MRI[22, 23]. The procedure of selecting the patients in these two groups and giving the 
diagnosis were performed by experienced spine surgeons who did not know the purpose of the study. No statistically significant differences in the variables age, gender, labor intensity and BMI were noted between the two groups $(P>0.05)$. The work has been reported in line with the STROCSS criteria[24].

\section{Patient Selection}

Patients in group 1 were included in the study if they had (a) symptomatic cervical spondylotic myelopathy, the thoracic and lumbar IDH. The diagnosis of cervical spondylotic myelopathy and of thoracic and lumbar IDH was conducted on the basis of clinical presentation, physical examination, radiography, electromyography, computerized tomography (CT) and/or MRI. (b) Absence of symptom relief despite adequate medical treatment.

The inclusion criteria for group 2 were the following: (1) The patients who underwent physical examination in the same period and were excluded for IVDD by spine MRI from 2009 to 2019[2]. The patients who were frequency-matched by age and gender with the patients of Group 1 (Table1).

The exclusion criteria for groups 1 and 2 were the following: history of spinal disorders, multiple IDHs, spondylolysis, spondylolisthesis, foraminal or central canal stenosis, spinal trauma, spondyloarthritis, primary osteoarthritis of the operated and/or contralateral joint, inflammatory joint disease, diabetes, coronary heart disease, cerebrovascular disease, hypertension, smoking and age lower than 18 years[16].

\section{The definition of symptomatic IDH}

\section{The cervical disc herniation [25]}

(1)Typical sensory radicular symptoms (pain or paresthesias) were always present. (2)Motor (weakness and atrophy), sensory (hypesthesia or dysesthesia), and reflex (diminution or absence of tendon reflexes) symptoms, if present, were confined to 1 dermatome and/or myotome that corresponded to pain and/or paresthesias; Positive signs: a positive Spurling's test, Eaten test and Hoffman sign. (3)Clinical symptoms and signs correlated with the level of root compression as a result of disc herniation and/or spondylotic lateral stenosis.

\section{The thoracic disc herniation [26]}

(1)Localized axial back pain and/or axial back pain with radiation into the lumbar spine; sensory impairment; (2) Special nerve root irritation signs: the pain can even mimic cardiac disease and/or present as abdominal and/or shoulder pain; (3) Neurologic deficit: paraparesis and monoparesis; spasticity and hyperreflexia; bladder dysfunction; (knee jerk or ankle reflex).

\section{The lumbar disc herniation [16]}

(1)Low back pain with unilateral or bilateral lower limb radicular pain; (2)Special nerve root irritation signs: straight leg raising test and strengthen test and/or femoral stretch test depending on the level of 
injury (3) Neurologic deficit: muscle weakness, numbness, and/or lack of the corresponding reflex (knee jerk or ankle reflex).

\section{Imaging diagnosis}

All the patients enrolled in the present study underwent the spine examination by $1.5 \mathrm{~T}$ MRI. Both T2 and T1-weighted images were combined and used to assess the IVDD from C1/2 to L5/S1 regions by an experienced spine surgeon who was blinded to the study. The degree of IVDD on MRI was based on the Pfirrmann grade system[25]. The criteria for grade 1 are the following: Its structure is homogeneous and bright white in color; The distinction of nucleus and anulus is clear; The signal intensity for the disc is hyperintense and isointense compared with the cerebrospinal fluid; The height of the intervertebral disc is normal. The criteria for grade 2 are the following: Its structure is nonhomogeneous in the absence and/or presence of horizontal bands; The distinction of the nucleus and anulus is clear; The signal intensity for the disc is hyperintense and isointense compared with the cerebrospinal fluid; The height of the intervertebral disc is normal. The criteria for grade 3 are as follows: Its structure is progressing to nonhomogeneous and gray in color; The distinction of nucleus and anulus is unclear; The signal intensity for the disc is intermediate; The height of the intervertebral disc is normal to slightly decreased. The criteria for grade 4 are the following: Its structure is progressing to nonhomogeneous and gray to black in color; The distinction of the nucleus and the anulus is lost; The signal intensity for the disc is intermediate to hypo-intense; The height of the intervertebral disc is normal to slightly decreased. The criteria for grade 5 are as follows: Its structure is progressing to nonhomogeneous and black in color; The distinction of the nucleus and anulus is lost; The signal intensity for the disc is hypointense; The disc space is collapsed[27-29].

\section{Blood examination}

All blood samples were collected in an identical manner between 07.30 and 08.30 a.m. following an overnight fast that started at 12.00 midnight. Biochemical analyses of blood samples were conducted on fresh specimens. Fasting blood samples were collected, and five milliliters of each sample was centrifuged at 4,000 rpm for $6 \mathrm{~min}$. The serum was extracted from the samples, and the concentrations of TC, TG, LDL-C, HDL-C, Apo Al, Apo B, Lp(a) were measured by an automatic biochemical analyser in an identical manner. The normal levels of the following indexes exhibited the following range TC from 0 to $5.2 \mathrm{mmol} / \mathrm{L}$; TG from 0 to $1.7 \mathrm{mmol} / \mathrm{L}$; LDL-C from 0 to $3.4 \mathrm{mmol} / \mathrm{L} ; \mathrm{HDL}-\mathrm{C}$ from 0.7 to $2.0 \mathrm{mmol} / \mathrm{L}$; ApoEA1 from 1 to $1.6 \mathrm{~g} / \mathrm{L} ; \mathrm{Lp}(\mathrm{a})$ from 0 to $30 \mathrm{mg} / \mathrm{mL}$; Apo $B$ from 0.6 to $1.1 \mathrm{~g} / \mathrm{L}$. The normal value of the ratio of Apo B/Apo Al was 0.87 for the male subjects, and 0.65 for the female subjects[30].

\section{Statistical analysis}

Continuous variables were expressed as the mean \pm standard deviation (SD) and analyzed with unpaired t-tests. Categorical variables were expressed as a percentage of the number and analyzed with a chisquare test. The normality analysis was carried out for the continuous variables. SPSS (Version 20.0) was used for all statistical analyses. A P value that was lower than 0.05 was regarded as statistically 
significant. Multivariate logistic regression was used to evaluate the effects of serum lipids on symptomatic IDH. The effect indicators were odds ratio (OR) and $95 \%$ confidence interval (CI). The data of continuous variables in the present study followed an ordinary normal distribution. The adjustment for multiple comparisons was conducted in the present study. A P value that was lower than $0.007(P<0.007)$ was considered statistically significant following the adjustment for multiple comparisons (Table 1 and Figure 2). The correlations analysis was carried out in the present study. A P value that was lower than $0.05(P<0.05)$ was considered statistically significant, A $P$ value that was lower than $0.01(P<0.01)$ was considered extremely statistically significant.

\section{Results}

\section{IDH patients exhibited significantly higher TC, TG, LDL, Apo B, Lp(a) and Apo B/Apo Al levels}

The serum concentrations of TC, TG, LDL-C, HDL-C, Apo Al, Apo B and $L p(a)$ were measured in all patients (Figure2). In Group 1 (IDH), the concentration levels of TG, total TC, LDL-C, HDL-C, Apo B and Lp(a) were $1.63 \pm 1.26 \mathrm{mmol} / \mathrm{L}, 4.50 \pm 1.48 \mathrm{mmol} / \mathrm{L}, 2.67 \pm 0.9 \mathrm{mmol} / \mathrm{L}, 1.16 \pm 0.29 \mathrm{mmol} / \mathrm{L}, 0.80 \pm 0.22 \mathrm{~g} / \mathrm{L}$ and $25.35 \pm 24.01 \mathrm{mg} / \mathrm{dl}$, respectively. In Group 2 (control group), the concentration levels of TC, TG, LDL-C, HDL-C, Apo Al, Apo B and Lp(a) were 4.23 $\pm 2.18 \mathrm{mmol} / \mathrm{L}, 1.4 \pm 0.91 \mathrm{mmol} / \mathrm{L}, 2.52 \pm 0.83 \mathrm{mmol} / \mathrm{L}, 1.21 \pm 0.36$ $\mathrm{mmol} / \mathrm{L}, 0.76 \pm 0.23 \mathrm{~g} / \mathrm{L}$ and $22.45 \pm 21.11 \mathrm{mg} / \mathrm{dl}$, respectively. The patients with symptomatic IDH indicated significantly higher levels of TG $(P=0.002), T C(P=0.00), L D L-C(P=0.00), A p o B(P=0.00), L p(a)$ $(P=0.006)$. No statistically significant differences were noted for the HDL-C $(P=0.125)$ and Apo Al $(P=0.326)$. The ratios of Apo B/Apo Al were higher in the symptomatic IDH comparing with control group $(0.78 \pm 0.33$ versus $0.71 \pm 0.25, P<0.01)$.

\section{The percentage of high-TC, high-TG, high-LDL, high-Apo B and high-Lp(a) were significantly increased in the IDH group}

The percentage of dyslipidemia incidence in the control and IDH group were further investigated in our study. The incidence of the high TC, high TG, high-LDL-C, low-HDL-C, Apo Al, Apo B and Lp(a) were $31.19 \%, 13.85 \%, 20.17 \%, 7.52 \%, 14.9 \%, 30.8 \%$ and $28.9 \%$, respectively, compared to $23.33 \%, 15.34 \%$, $14.8 \%, 5.98 \%, 13.6 \%, 21.76 \%$ and $51.4 \%$ that was noted in the control group, respectively (Table 3 ). The incidence of the high- TC, high-TG and high-LDL-C, Apo B and Lp(a) were significantly higher in the IDH group compared with the control group $(P=0.000, P=0.00, P=0.02, P=0.000, P=0.000$ respectively). No statistically significant differences were noted with regard to HDL-C $(P=0.189)$ and $\operatorname{Apo~Al}(P=0.412)$.

\section{The association between serum lipid abnormalities and the degree of IVDD}

To further investigate correlation for the incidence of a symptomatic IDH with an elevated LDL-C, TC, TG, $\mathrm{Lp}(\mathrm{a})$, Apo B and Apo B/Apo Al, the correlation analysis was conducted between serum lipid abnormalities and the degree of IVDD (Pfirrmann grade). As shown in Figure 3, the correlation between elevated LDL-C, TC, TG, Apo $B$, $L p(a)$ and incidence of IDH were significant $\left(R^{2} L D L=0.017, P<0.001\right.$; $\left.R^{2} T C=0.004, P<0.004 ; R^{2} T G=0.015, P<0.001 ; R^{2} A p o B=0.004, P<0.001 ; R^{2} L P(a)=0.021, P<0.008\right)$. These 
results suggested that patients with higher LDL-C, TC, TG, Lp(a), Apo B and Apo B/Apo Al levels are closely related with disc herniation.

\section{Hyperlipidaemia did not affect the degenerated segment of the intervertebral disc}

The categorical data of the patients with disc herniation were analyzed in order to examine the associations between hyperlipidaemia and the disc segment in the intervertebral disc group. The hyperlipidaemia group $(n=689)$ exhibited the following percentages of degenerated segments in the cervical, thoracic and lumbar regions: $13.9 \%, 1.3 \%$ and $84.8 \%$, respectively. In contrast to the hyperlipidemic samples, the normal serum lipids group exhibited incidences of $18.8 \%, 1.3 \%$ and $79.9 \%$ that corresponded to the cervical, thoracic and lumbar regions, respectively $(n=229)$. No significant differences in the herniation segments were noted between these two groups $(p=0.201)$ (Figure 4).

The categorical data were further analyzed in order to identify the association between the serum lipid levels and the segment of disc herniation in the cervical and lumbar regions. Considering the small sample of affected segment in the thoracic disc herniation, we only analyzed the serum lipid levels and segment of disc herniation in the cervical and lumbar regions.

The values of the total segment in the cervical, thoracic and lumbar regions were 137, 12 and 769 respectively. No significant differences were noted between serum lipid levels in the $\mathrm{C} 3-\mathrm{C} 4(\mathrm{P}=0.282)$ and C5-6 ( $P=0.373)$ segments with regard to TC levels (Figure 5A). Similarly, no significant differences were observed in the $C 3-C 4(P=0.108)$ and $C 5-6$ segments with regard to $L D L-C$ levels $(P=0.254)$ (Figure $5 C)$. With regard to the levels of Apo B, the C5-6 segment in the hyperlipidaemia group (31.9\%) was higher than that of the normal group (24.2\%), although no significant differences were noted (Figure 5D, $P=0.2$ ). With regard to the levels of ApoA (Figure 5E), Lp(a) (Figure 5F) and triglycerides (TG) (Figure 5B), the distribution herniation segment in the hyperlipidaemia and control groups exhibited similar trends both in the lumbar and cervical segments. However, we do not get the significance statistic in our study. Comparing with cervical and lumbar IDH, the incident of thoracic IDH is quite low. The relatively small sample size in our study may contribute to the no-significance result.

\section{Patients with elevated LDL-C, TC, TG, Lp(a), Apo B and Apo B/Apo Al levels exhibited a higher risk of disc herniation}

To further identify risk for the incidence of a symptomatic IDH with an elevated LDL-C, TC, TG, Lp(a), Apo $\mathrm{B}$ and Apo B/Apo Al, multivariate logistic regression analysis was performed. As shown in Table 4, the odds ratios (ORs) for the incidence of a symptomatic IDH with an elevated LDL-C, TC, TG, Lp(a), Apo B and Apo B/Apo Al were 1.583 (Cl, 1.427-1.796), 1.74(Cl, 1.282-2.365), 1.62 (Cl, 1.295-2.023), 1.58(Cl, $1.255-1.975), 1.49(\mathrm{Cl}, 1.346-1.661)$ and $1.39(\mathrm{Cl}, 1.254-1.595)$, respectively $(\mathrm{P}<0.01)$. These results suggested that patients with higher LDL-C, TC, TG, Lp(a), Apo B and Apo B/Apo Al levels exhibited a higher risk of disc herniation.

\section{Discussion}


The association between serum lipid and IVDD related disease has been examined by a multitude of studies[12-16]. Elevated levels of TC, LDL-C and TG have been shown to be associated with sciatica[12], back pain and/or disc herniation[13-16, 28]. In agreement with these results, our data showed IDH patients exhibited significantly higher TC, TG, LDL levels and the percentage of high-TC, high-TG, highLDL were significantly increased in the IDH group.

Results from several clinical prospective studies indicate that the Apo B/ApoA1 ratio is a simple, accurate risk factor for cardiovascular, osteoarthritis, rheumatoid arthritis, metabolic syndrome disease[19-21, 27]. A Swedish prospective study showed that the Apo B/ApoA1 was a robust and specific marker in predicting the risk of coronary disease and ischemic events[19]. Recently, more and more study found that the Apo B/ApoA1 ratio is the independent risk factor among smoking, hypertension, abdominal obesity, diabetes, psychosocial stress, and acute myocardial infarction [20, 21, 27, 31, 32]. However, as aged related degenerated disease, whether any association between Apo Al, Apo B, Apo B/Apo Al and $\mathrm{Lp}(\mathrm{a})$ levels and symptomatic IDH are still unclear.

In the present study, the relationship between Apo Al, Apo B, Lp(a) and symptomatic IDH were examined for the first time. As a result, the levels of $A p o B$ and $L p(a)$ were shown to positively associate with the incidence of symptomatic IDH. Apo B serves as an essential structural component of chylomicrons, VLDL, intermediate density lipoprotein (IDL) and LDL-C. For the LDL-C, Apo B is a ligand that facilitates cholesterol delivery to the tissues and promotes cholesterol accumulation in the arterial tissue. However, Apo Al is a structural component of HDL-C that facilitates the uptake of cholesterol from the peripheral cells. Moreover, Apo Al is an activator of the lecithin-cholesterol acyltransferase, a key enzyme helping transport of cholesterol to the liver for digesting. Thus, the plasma concentration levels of these Apo $\mathrm{B} / \mathrm{Apo} \mathrm{Al}$ may reflect cholesterol transport ability to the peripheral tissues and indicate a balance between atherogenic and anti-atherogenic factors[31, 32]. Elevated levels of the plasma concentrations of Apo B and increased proportion of the Apo B/Apo Al ratio were observed in symptomatic IDH patients suggested a prominent cholesterol transport to the peripheral tissues including IVD in these patients. $\mathrm{Lp}(\mathrm{a})$ is composed of a lipoprotein particle, having the similar protein and lipid proportion with LDL-C. Various studies have shown that high levels of $L p(a)$ in plasma can be a risk factor for cardiovascular disease, osteoarthritis, and rheumatoid arthritis[33]. The plasma concentrations of $L p(a)$ are associated with a genetic basis and race/ethnicity [34]. At the same time, the plasma Lp(a) levels may be affected by age, drugs, diabetes, diet and so on. In the present study, it was shown that the level of Lp(a) was increased in the intervertebral dis herniation group. The current study is the first study to report this finding in the field of symptomatic intervertebral disc herniation.

The precise pathophysiologic mechanism underlying the connection between serum lipid levels and lumbar disc herniation remains unclear. The increasing levels of TC, TG, LDL-C, Apo B and Lp(a) in IDH patients could be mainly caused by several reasons. Firstly, the IVD is a poorly vascularized region, whose nutritional supply is through the blood capillary penetration of endplate cartilage and the aunnual fibrosis[29]. High levels of serum cholesterol[28], triglycerides [28, 35-37], LDL-C[38], Apo B and Lp(a) are considered contribute to atherosclerosis. The presence of atherosclerotic will inhibit the vascular supply 
to the poorly vascularized IVD and induced IVDD/IDH[39]. In agreement with our hypothesis, the association between symptomatic IDH and atherosclerosis were identified by a lot of studies. A study that included 86 people concluded that atherosclerosis in the abdominal aorta and notably stenosis of the ostia of segmental arteries may play a vital role in symptomatic IDH[40]. Another 25-year follow-up study showed the calcific atherosclerotic deposits in the posterior wall of the abdominal aorta increased the risk of disc herniation and back pain[41]. Secondly, the activated inflammatory cells induced by high serum lipid levels may comprise an important pathway in the development of symptomatic IDH for hyperlipidaemia patients. The activation of cytokines plays a significant role in the development of IVDD/IDH[39, 42]. Previous studies have reported that pro-inflammatory cytokines were closely associated with serum lipid levels[5, 43]. Therefore, the increased serum lipid levels may enhance the inflammatory response and/or the basic level of systemic inflammation, which can in turn contribute to disc herniation[6]. Thirdly, the oxidized low-density lipoprotein (oxLDL) and the increased expression of lectin-like oxidized low-density lipoprotein receptor 1 (LOX-1) that are caused by dyslipidaemia may also be involved in the development of symptomatic IDH. Our previous study suggested[44] that the levels of the oxLDL and LOX-1 were positively correlated with the extent of IDH. The mechanism of action involved the increase in the expression of MMP3 that was induced by LOX-1. This in turn caused the oxLDL to significantly reduce the viability of human nucleus pulpous. The production of oxLDL usually originates from LDL-C that is oxidized under oxidative stress conditions. Therefore, the elevated LDL-C levels can increase level of oxLDL/LOX-1 and accelerate IVDD.

As is known, elevated levels of TC, TG, LDL-C, Apo B, Lp(a) and reduced HDL-C level are atherogenic lipid marker. The management of cardiovascular disease has traditionally focused on reducing serum lipid levels[45], In this study, we found that elevated serum lipid levels were significantly correlated with IDH and High serum lipid levels predicted a higher incidence of IDH. This association opens the way for a new approach to reducing the risk of IDH/IVDD disease by controlling serum lipid levels.

There were several limitations in the present study: Firstly, no data were provided regarding the levels of very low-density lipoproteins (VLDL). Secondly, this was a retrospective case-control study, thus the causal relationships between serum lipid components and symptomatic IDH remain unclear. These associations can only be ascertained by a follow-up study that will examine the incidence and/or progression rates of symptomatic IDH cases of the same cohort. In the current study, we do not determine whether elevated lipid levels directly give rise to IDH/IVDD, or the elevation of lipids causes other disorders, which in turn lead to IDH/IVDD. In order to prove cause and effect relationships and to find effective treatments for IVDD, a large longitudinal follow-up observations and intervention studies is needed[16]. In the following investigations, we will enlarge the sample size to illustrate the potential association between serum lipids and disc herniation.

\section{Conclusion}

The level of TC, TG, LDL-C, Apo B (Apo B/Apo Al) and Lp (a) were positively correlated with the incidence of symptomatic IDH. However, hyperlipidaemia did not affect the degenerated segment of the 
intervertebral disc. This association provides novel evidence regarding the reduction of the risk of symptomatic IDH disease by the control of the serum lipid levels.

\section{Declarations}

Ethical Approval and consent to participate: All procedures performed in studies involving human participants were approved prospectively by the authors' human subjects Institutional Review Board.

Informed consentロAll of the participants consented to participate in this study.

\section{Availability of data and materials}

The datasets used and/or analyzed during the current study are available from the corresponding author on reasonable request.

\section{Consent for publication}

Not applicable

\section{Competing interests}

The authors declare that they have no competing interests

\section{Funding}

This paper was supported by grants from National Natural Science Foundation of China (81672199), National Natural Science Foundation of China (81371994); National Natural Science Foundation of China (81572181), Natural Science Foundation of Shanghai (19ZR1441700).

\section{Author contributions}

$\mathrm{XL}$ and $\mathrm{FC}$ interpreted the data and wrote the initial draft of the manuscript. $\mathrm{XL}, \mathrm{TW}, \mathrm{SG}, \mathrm{JH}, \mathrm{QF}, \mathrm{YP}, \mathrm{HZ}$, DW, and LJ conceived, supervised the study, and wrote the manuscript. QC and LL provided critical suggestions during the study. All authors read and approved the final manuscript.

\section{Acknowledgement}

Research reported in this publication was supported by the National Natural Science Foundation of China (81672199), National Natural Science Foundation of China (81371994); National Natural Science Foundation of China (81572181), Natural Science Foundation of Shanghai (19ZR1441700). The authors acknowledge the patients who agreed to involve in this study.

\section{References}

1. Andersson GB: Epidemiological features of chronic low-back pain. Lancet 1999, 354(9178):581-585. 
2. Luoma K, Riihimaki H, Luukkonen R, Raininko R, Viikari-Juntura E, Lamminen A: Low back pain in relation to lumbar disc degeneration. Spine (Phila Pa 1976) 2000, 25(4):487-492.

3. Juniper M, Le TK, Mladsi D: The epidemiology, economic burden, and pharmacological treatment of chronic low back pain in France, Germany, Italy, Spain and the UK: a literature-based review. Expert Opin Pharmacother 2009, 10(16):2581-2592.

4. Wade KR, Robertson PA, Thambyah A, Broom ND: How healthy discs herniate: a biomechanical and microstructural study investigating the combined effects of compression rate and flexion. Spine (Phila Pa 1976) 2014, 39(13):1018-1028.

5. Hussein Al, Jackman TM, Morgan SR, Barest GD, Morgan EF: The intravertebral distribution of bone density: correspondence to intervertebral disc health and implications for vertebral strength. Osteoporos Int 2013, 24(12):3021-3030.

6. Modic MT, Ross JS: Lumbar degenerative disk disease. Radiology 2007, 245(1):43-61.

7. Hirayama J, Yamagata M, Ogata S, Shimizu K, Ikeda Y, Takahashi K: Relationship between low-back pain, muscle spasm and pressure pain thresholds in patients with lumbar disc herniation. Eur Spine $J$ 2006, 15(1):41-47.

8. Mehta JL, Chen J, Hermonat PL, Romeo F, Novelli G: Lectin-like, oxidized low-density lipoprotein receptor-1 (LOX-1): a critical player in the development of atherosclerosis and related disorders. Cardiovasc Res 2006, 69(1):36-45.

9. Li X, Han Y, Di Z, Cui J, Pan J, Yang M, Sun G, Tan J, Li L: Percutaneous endoscopic lumbar discectomy for lumbar disc herniation. J Clin Neurosci 2016, 33:19-27.

10. Li X, Hu Z, Cui J, Han Y, Pan J, Yang M, Tan J, Sun G, Li L: Percutaneous endoscopic lumbar discectomy for recurrent lumbar disc herniation. Int J Surg 2016, 27:8-16.

11. Li X, Yang S, Han L, Mao K, Yang S: Ciliary IFT80 is essential for intervertebral disc development and maintenance. FASEB J 2020, 34(5):6741-6756.

12. Leino-Arjas P, Kauppila L, Kaila-Kangas L, Shiri R, Heistaro S, Heliovaara M: Serum lipids in relation to sciatica among Finns. Atherosclerosis 2008, 197(1):43-49.

13. Hangai M, Kaneoka K, Kuno S, Hinotsu S, Sakane M, Mamizuka N, Sakai S, Ochiai N: Factors associated with lumbar intervertebral disc degeneration in the elderly. Spine $J$ 2008, 8(5):732-740.

14. Leino-Arjas P, Kaila-Kangas L, Solovieva S, Riihimaki H, Kirjonen J, Reunanen A: Serum lipids and low back pain: an association? A follow-up study of a working population sample. Spine (Phila Pa 1976) 2006, 31(9):1032-1037.

15. Longo UG, Denaro L, Spiezia F, Forriol F, Maffulli N, Denaro V: Symptomatic disc herniation and serum lipid levels. Eur Spine J 2011, 20(10):1658-1662.

16. Zhang Y, Zhao Y, Wang M, Si M, Li J, Hou Y, Jia J, Nie L: Serum lipid levels are positively correlated with lumbar disc herniation-a retrospective study of 790 Chinese patients. Lipids Health Dis 2016, $15: 80$. 
17. Heuch I, Heuch I, Hagen K, Zwart JA: Do abnormal serum lipid levels increase the risk of chronic low back pain? The Nord-Trondelag Health Study. PLoS One 2014, 9(9):e108227.

18. Keser N, Celikoglu E, Is M, Ilgezdi ZD, Sunar B, Aydin YS, Kevenk AU, Gurer B, Ramazanoglu AF, Keser $\mathrm{N}$ : Is there a relationship between blood lipids and lumbar disc herniation in young Turkish adults? Arch Med Sci Atheroscler Dis 2017, 2(1):e24-e28.

19. Yin Q, Chen X, Li L, Zhou R, Huang J, Yang D: Apolipoprotein B/apolipoprotein A1 ratio is a good predictive marker of metabolic syndrome and pre-metabolic syndrome in Chinese adolescent women with polycystic ovary syndrome. J Obstet Gynaecol Res 2013, 39(1):203-209.

20. Zegarra-Mondragon S, Lopez-Gonzalez R, Martin-Martinez MA, Garcia-Gomez C, Sanchez-Alonso F, Gonzalez-Juanatey C, Manrique Arija S, Bonilla Hernan G, Martinez Pardo S, Ruibal Escribano A et al: Association of apolipoprotein B/apolipoprotein A1 ratio and cardiovascular events in rheumatoid arthritis: results of the CARMA study. Clin Exp Rheumatol 2020, 38(4):662-669.

21. Zhan X, Chen Y, Yan C, Liu S, Deng L, Yang Y, Qiu P, Pan D, Zeng B, Chen Q: Apolipoprotein $\mathrm{B}$ /apolipoprotein $\mathrm{A} 1$ ratio and mortality among incident peritoneal dialysis patients. Lipids Health Dis 2018, 17(1):117.

22. Pfirrmann CW, Metzdorf A, Zanetti M, Hodler J, Boos N: Magnetic resonance classification of lumbar intervertebral disc degeneration. Spine (Phila Pa 1976) 2001, 26(17):1873-1878.

23. Teraguchi M, Yoshimura N, Hashizume H, Muraki S, Yamada H, Oka H, Minamide A, Ishimoto $Y$, Nagata K, Kagotani R et al: Metabolic Syndrome Components Are Associated with Intervertebral Disc Degeneration: The Wakayama Spine Study. PLoS One 2016, 11(2):e0147565.

24. Agha RA, Borrelli MR, Vella-Baldacchino M, Thavayogan R, Orgill DP, Group S: The STROCSS statement: Strengthening the Reporting of Cohort Studies in Surgery. Int J Surg 2017, 46:198-202.

25. Bednarik J, Kadanka Z, Dusek L, Novotny O, Surelova D, Urbanek I, Prokes B: Presymptomatic spondylotic cervical cord compression. Spine (Phila Pa 1976) 2004, 29(20):2260-2269.

26. Babashahi A, Taheri M, Rabiee P: Spontaneous Resolution of Symptomatic Thoracic Spine Calcified Disc Herniation: A Case Report and Literature Review. Iran J Med Sci 2019, 44(3):251-256.

27. Sanchez-Enriquez S, Torres-Carrillo NM, Vazquez-Del Mercado M, Salgado-Goytia L, RangelVillalobos $\mathrm{H}$, Munoz-Valle JF: Increase levels of apo-A1 and apo B are associated in knee osteoarthritis: lack of association with VEGF-460 T/C and $+405 \mathrm{C} / \mathrm{G}$ polymorphisms. Rheumatol Int 2008, 29(1):63-68.

28. Hemingway H, Shipley M, Stansfeld S, Shannon H, Frank J, Brunner E, Marmot M: Are risk factors for atherothrombotic disease associated with back pain sickness absence? The Whitehall II Study. $J$ Epidemiol Community Health 1999, 53(4):197-203.

29. Kauppila LI: Prevalence of stenotic changes in arteries supplying the lumbar spine. A postmortem angiographic study on 140 subjects. Ann Rheum Dis 1997, 56(10):591-595.

30. Zhong L, Li Q, Jiang Y, Cheng D, Liu Z, Wang B, Luo R, Cheng Q, Qing H: The ApoB/ApoA1 ratio is associated with metabolic syndrome and its components in a Chinese population. Inflammation 2010, 33(6):353-358. 
31. Timur H, Daglar HK, Kara O, Kirbas A, Inal HA, Turkmen GG, Yilmaz Z, Elmas B, Uygur D: A study of serum Apo A-1 and Apo B-100 levels in women with preeclampsia. Pregnancy Hypertens 2016, 6(2):121-125.

32. Miyanishi K, Yamamoto T, Irisa T, Noguchi Y, Sugioka Y, Iwamoto Y: Increased level of apolipoprotein B/apolipoprotein A1 ratio as a potential risk for osteonecrosis. Ann Rheum Dis 1999, 58(8):514-516.

33. Cesur M, Ozbalkan Z, Temel MA, Karaarslan Y: Ethnicity may be a reason for lipid changes and high Lp(a) levels in rheumatoid arthritis. Clin Rheumatol 2007, 26(3):355-361.

34. Seriolo B, Accardo S, Fasciolo D, Bertolini S, Cutolo M: Lipoproteins, anticardiolipin antibodies and thrombotic events in rheumatoid arthritis. Clin Exp Rheumatol 1996, 14(6):593-599.

35. Stairmand JW, Holm S, Urban JP: Factors influencing oxygen concentration gradients in the intervertebral disc. A theoretical analysis. Spine (Phila Pa 1976) 1991, 16(4):444-449.

36. Boullart AC, de Graaf J, Stalenhoef AF: Serum triglycerides and risk of cardiovascular disease. Biochim Biophys Acta 2012, 1821(5):867-875.

37. Reiner Z: Hypertriglyceridaemia and risk of coronary artery disease. Nat Rev Cardio/ 2017, 14(7):401411.

38. Allaire J, Vors C, Couture P, Lamarche B: LDL particle number and size and cardiovascular risk: anything new under the sun? Curr Opin Lipido/ 2017, 28(3):261-266.

39. Kauppila LI, Penttila A, Karhunen PJ, Lalu K, Hannikainen P: Lumbar disc degeneration and atherosclerosis of the abdominal aorta. Spine (Phila Pa 1976) 1994, 19(8):923-929.

40. Igarashi A, Kikuchi S, Konno S, Olmarker K: Inflammatory cytokines released from the facet joint tissue in degenerative lumbar spinal disorders. Spine (Phila Pa 1976) 2004, 29(19):2091-2095.

41. Kauppila LI, McAlindon T, Evans S, Wilson PW, Kiel D, Felson DT: Disc degeneration/back pain and calcification of the abdominal aorta. A 25-year follow-up study in Framingham. Spine (Phila Pa 1976) 1997, 22(14):1642-1647; discussion 1648-1649.

42. Dagistan Y, Cukur S, Dagistan E, Gezici AR: Importance of IL-6, MMP-1, IGF-1, and BAX Levels in Lumbar Herniated Disks and Posterior Longitudinal Ligament in Patients with Sciatic Pain. World Neurosurg 2015, 84(6):1739-1746.

43. Studer RK, Vo N, Sowa G, Ondeck C, Kang J: Human nucleus pulposus cells react to IL-6: independent actions and amplification of response to IL-1 and TNF-alpha. Spine (Phila Pa 1976) 2011, 36(8):593599.

44. Li X, Wang X, Hu Z, Chen Z, Li H, Liu X, Yong ZY, Wang S, Wei Z, Han Y et al: Possible involvement of the oxLDL/LOX-1 system in the pathogenesis and progression of human intervertebral disc degeneration or herniation. Sci Rep 2017, 7(1):7403.

45. Schaefer EJ, Asztalos BF: The effects of statins on high-density lipoproteins. Curr Atheroscler Rep 2006, 8(1):41-49.

\section{Tables}


Due to technical limitations, table 1, 2, 3 and 4 is only available as a download in the Supplemental Files section.

\section{Figures}

Patients (control group) who underwent physical examination and accepted the spine MRI scan during the same period were matched with the baseline of the symptomatic intervertebral disc herniation group as much as possible: $\mathrm{n}=921$.

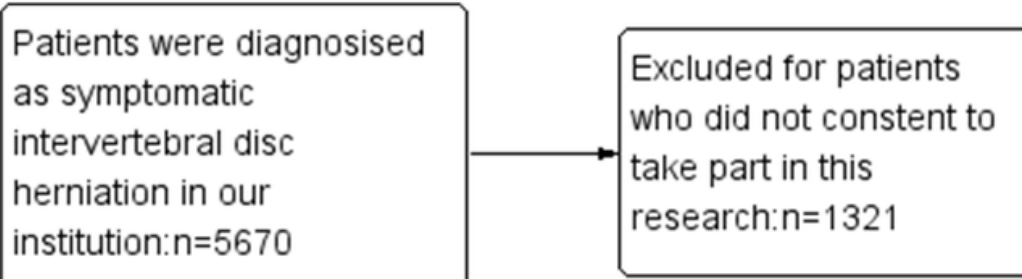

Patients were diagnosised as symptomatic intervertebral disc institution: $n=5670$

Excluded for patients owing to fail to meet included criteria: $n=3431$

\section{Figure 1}

The flow-chart of including and excluding 
Patients (control group) who underwent physical examination and accepted the spine MRI scan during the same period were matched with the baseline of the symptomatic intervertebral disc herniation group as much as possible: $\mathrm{n}=921$.

\begin{tabular}{|l|l|}
\hline $\begin{array}{l}\text { Patients were diagnosised } \\
\text { as symptomatic } \\
\text { intervertebral disc } \\
\text { herniation in our } \\
\text { institution: } n=5670\end{array}$ & $\begin{array}{l}\text { Excluded for patients } \\
\text { who did not constent to } \\
\text { take part in this } \\
\text { research: } n=1321\end{array}$ \\
\hline
\end{tabular}

Excluded for patients owing to fail to meet included criteria: $n=3431$

\section{Figure 1}

The flow-chart of including and excluding 


\section{Control group}

\section{Symptomatic intervertebral disc herniation group}

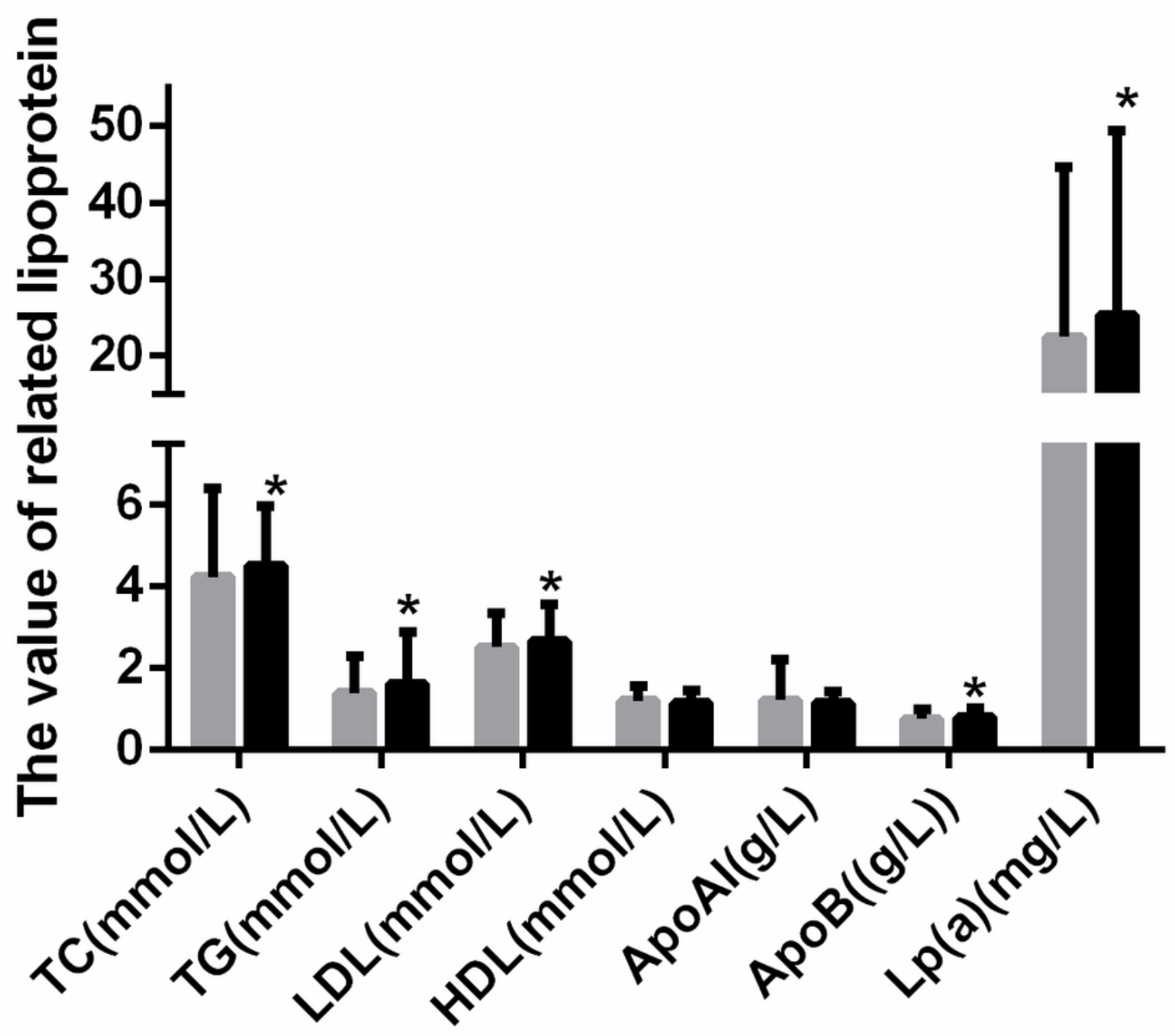

Figure 2

IDH patients exhibited significantly higher TC, TG, LDL, Apo B, Lp(a) and Apo B/Apo Al levels All data are reported as the mean \pm s.d. Statistical significance was determined by one-way ANOVA and Student's ttest. $* \mathrm{P}<.05$. NS $=$ not statistically significant. 


\section{Control group}

\section{Symptomatic intervertebral disc herniation group}

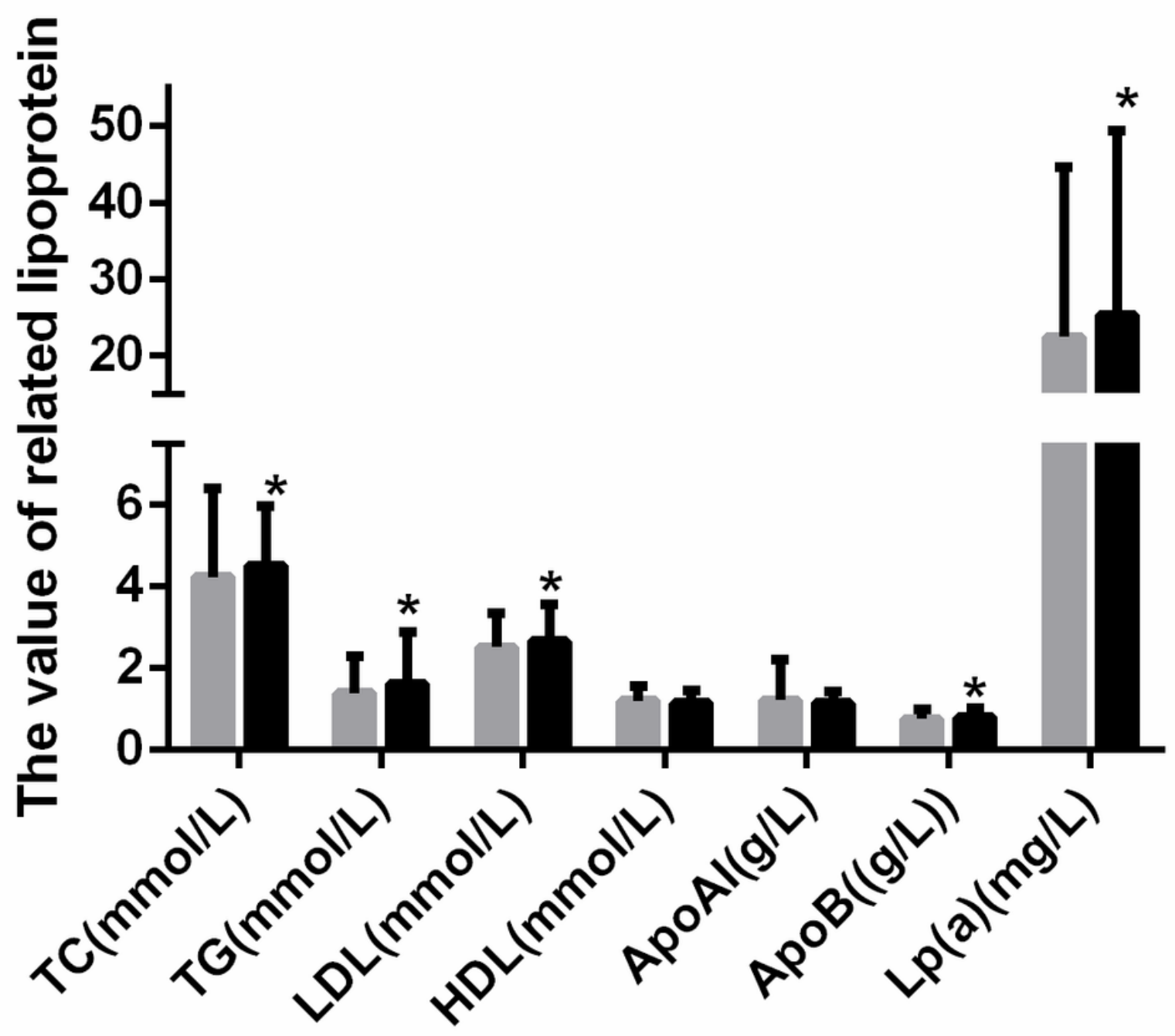

Figure 2

IDH patients exhibited significantly higher TC, TG, LDL, Apo B, Lp(a) and Apo B/Apo Al levels All data are reported as the mean \pm s.d. Statistical significance was determined by one-way ANOVA and Student's ttest. $* \mathrm{P}<.05$. NS $=$ not statistically significant. 
A

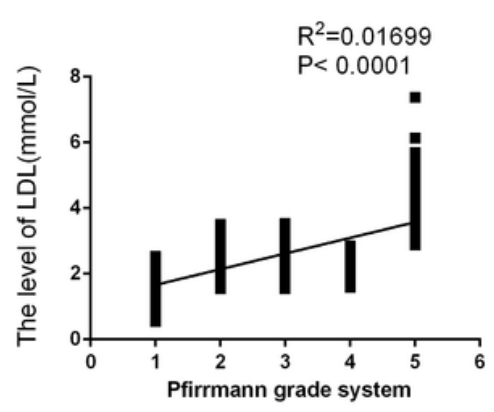

$\mathrm{D}$

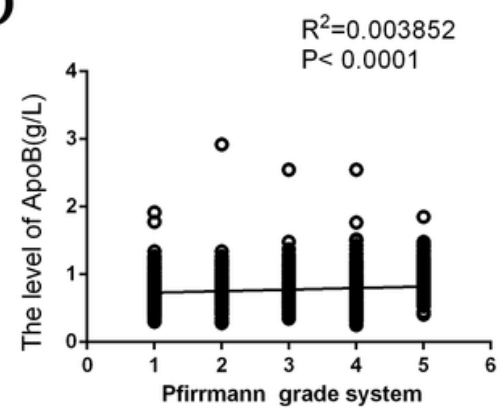

B

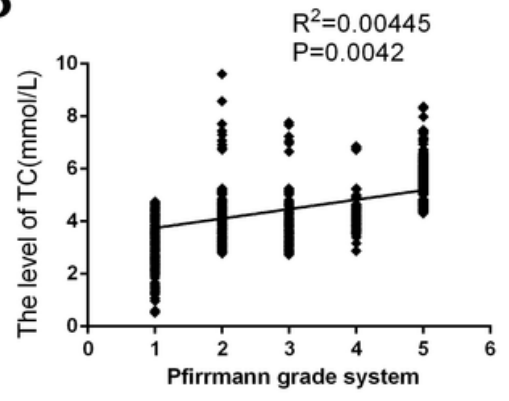

$\mathrm{E}$

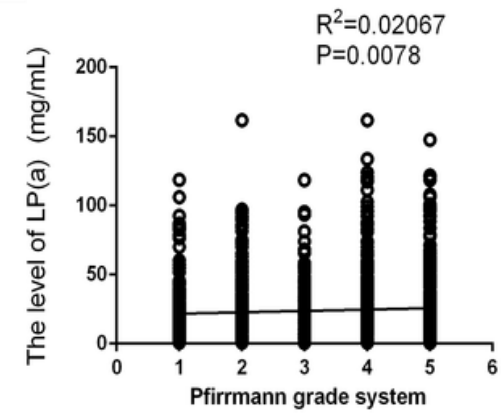

$\mathrm{C}$

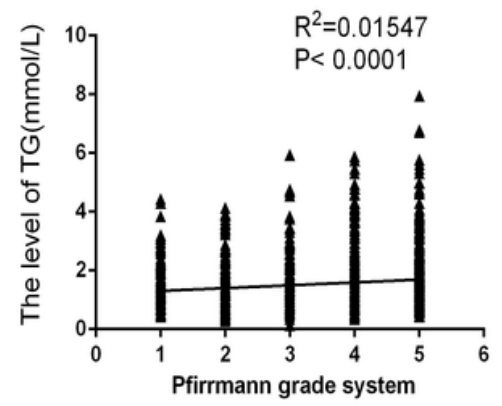

\section{Figure 3}

The association between serum lipid abnormalities and the grade of IVDD The correlation analysis between serum LDL (A), TC (B), TG (C), Apo B (D), LP(a) (E) and the grade of IVDD evaluated by pfirrmann grade system. 
A

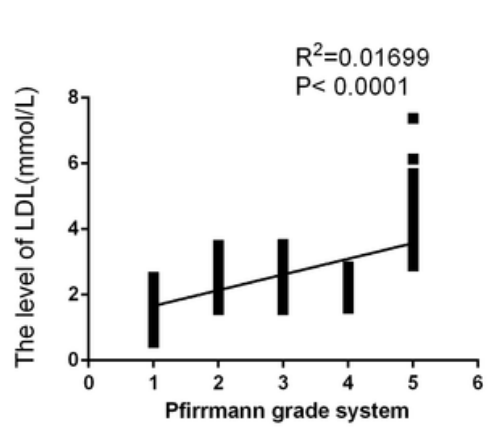

$\mathrm{D}$

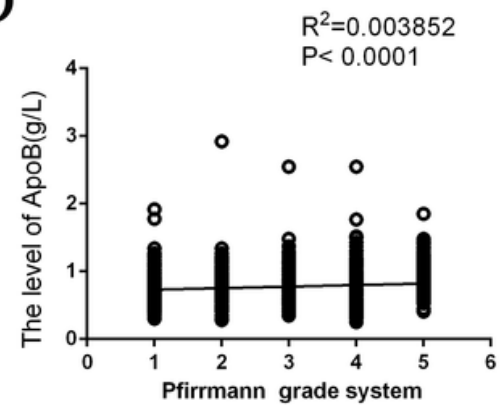

$\mathrm{B}$

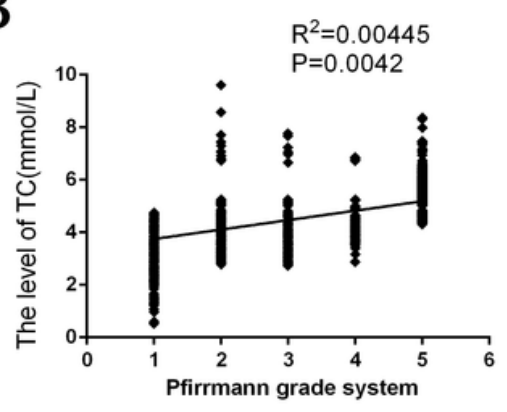

$\mathrm{E}$

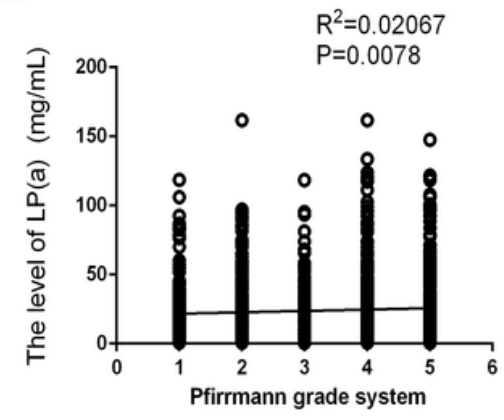

$\mathrm{C}$

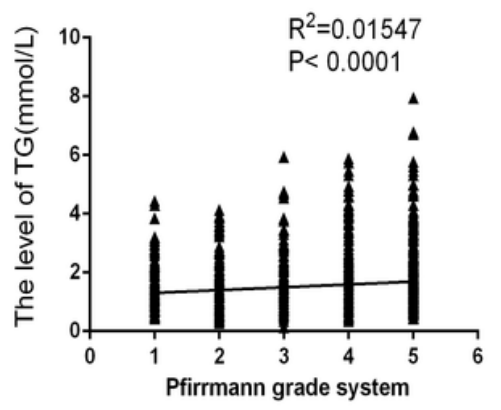

Figure 3

The association between serum lipid abnormalities and the grade of IVDD The correlation analysis between serum LDL (A), TC (B), TG (C), Apo B (D), LP(a) (E) and the grade of IVDD evaluated by pfirrmann grade system.

\section{The relationship between lipidaemia and affected segment}

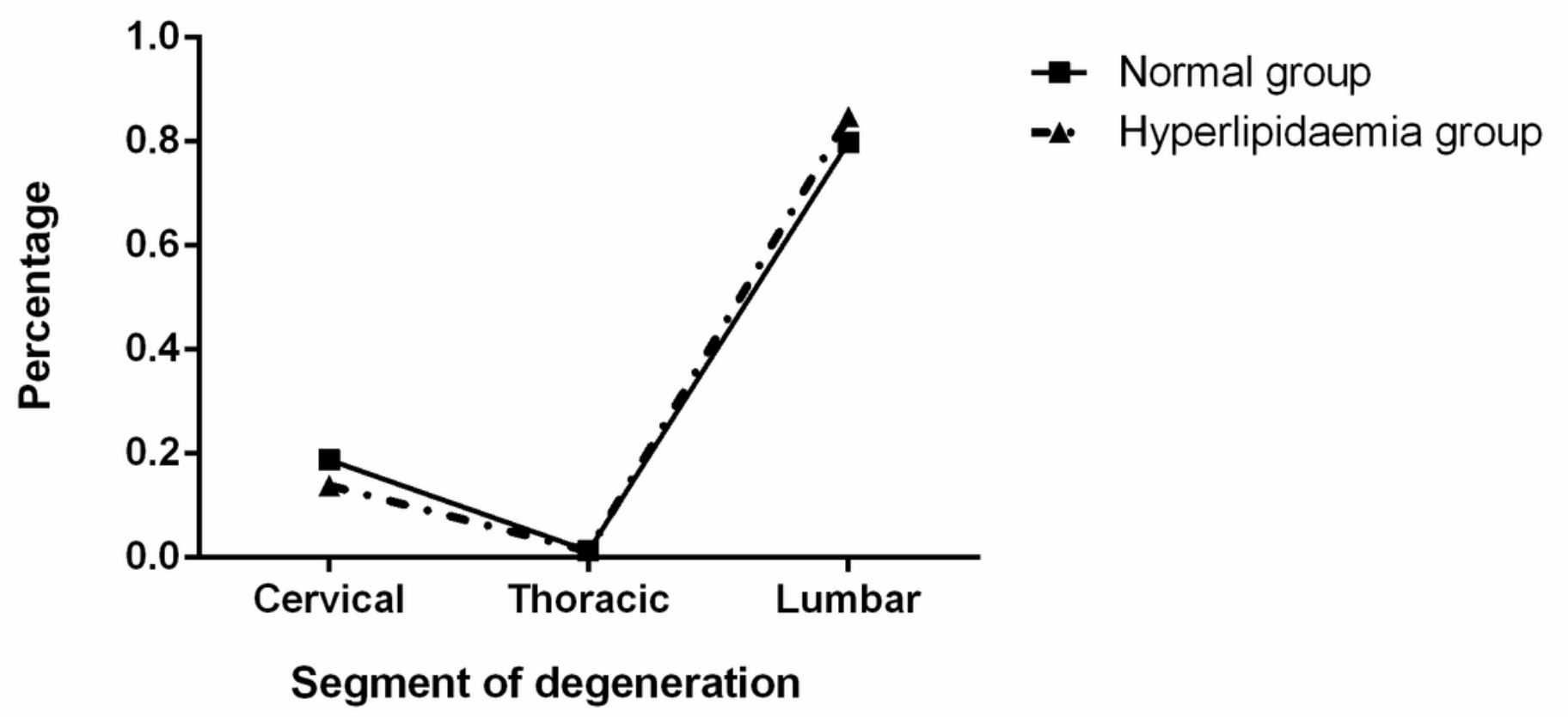


Figure 4

Hyperlipidaemia did not affect the segment of degenerated intervertebral disc

The relationship between lipidaemia and affected segment

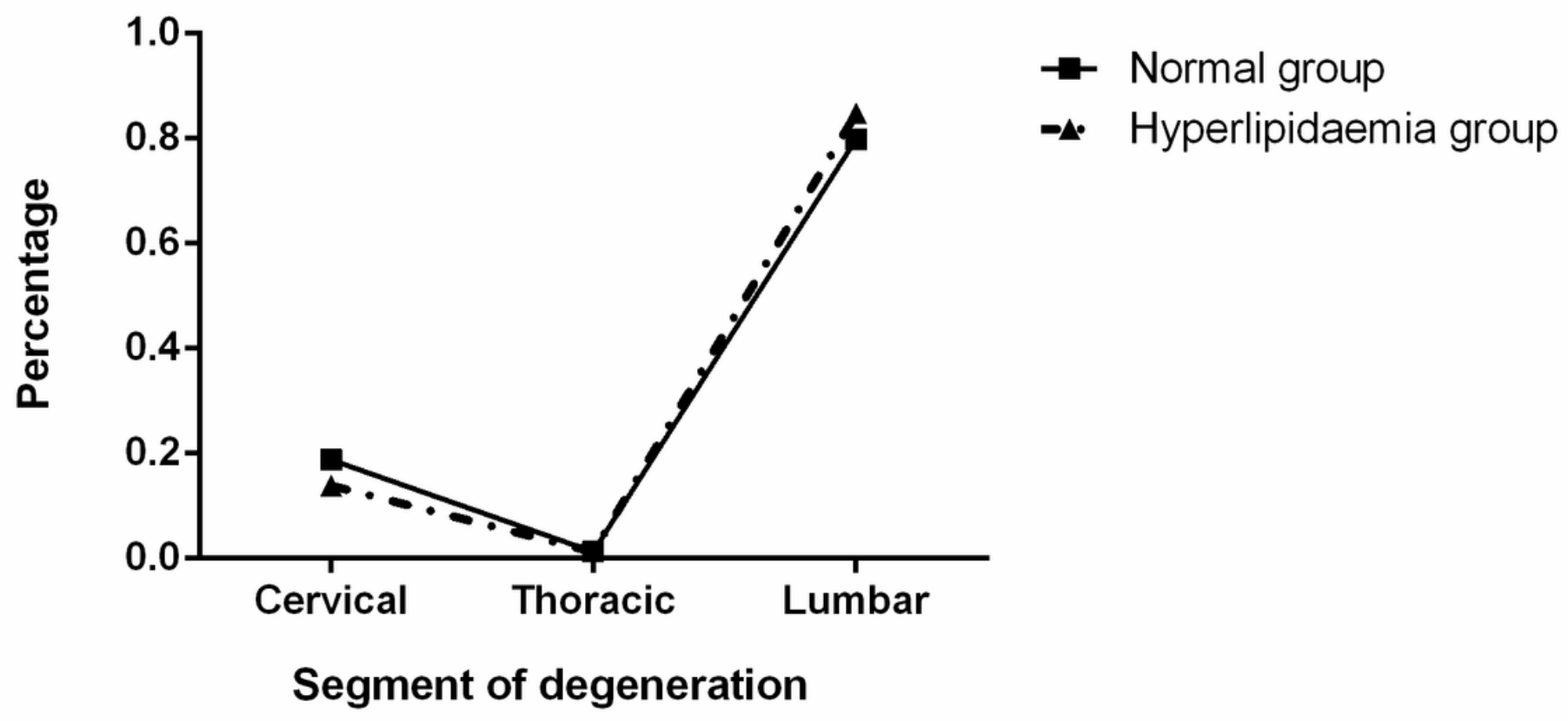

Figure 4

Hyperlipidaemia did not affect the segment of degenerated intervertebral disc 
A

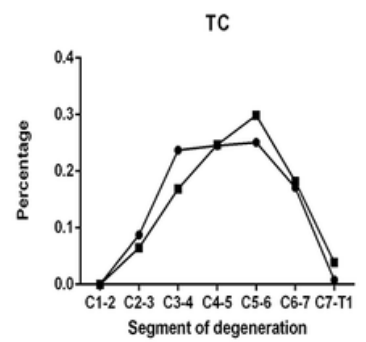

$\mathrm{C}$

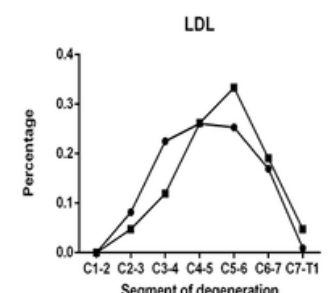

$\mathrm{E}$

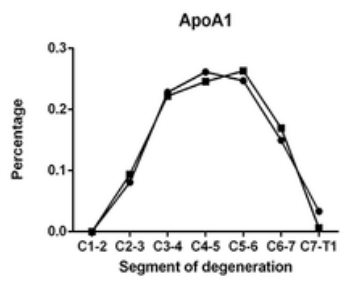

TC

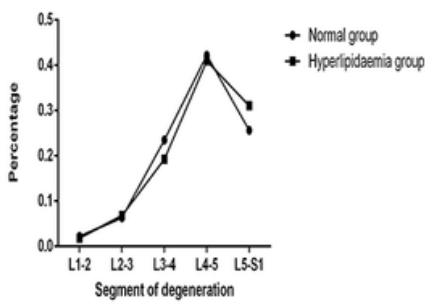

LDL
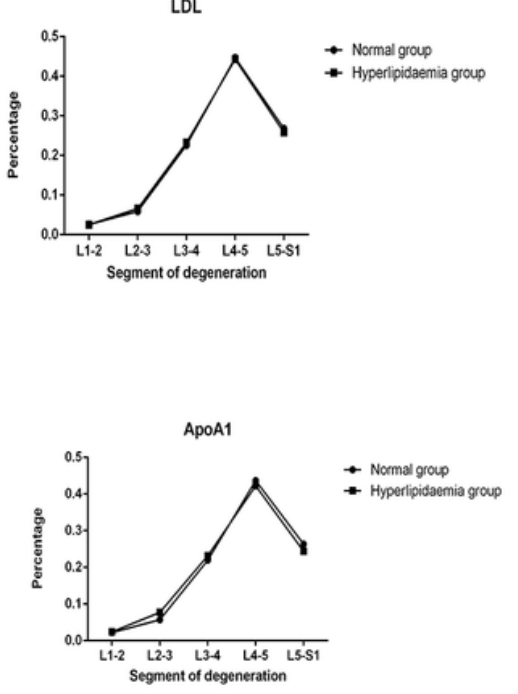

B
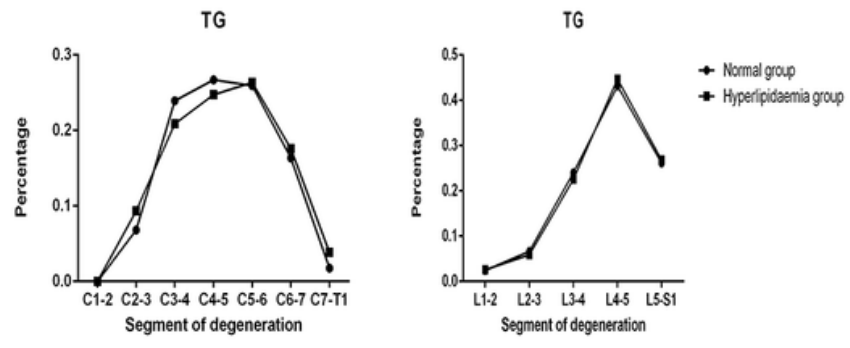

$\mathrm{D}$
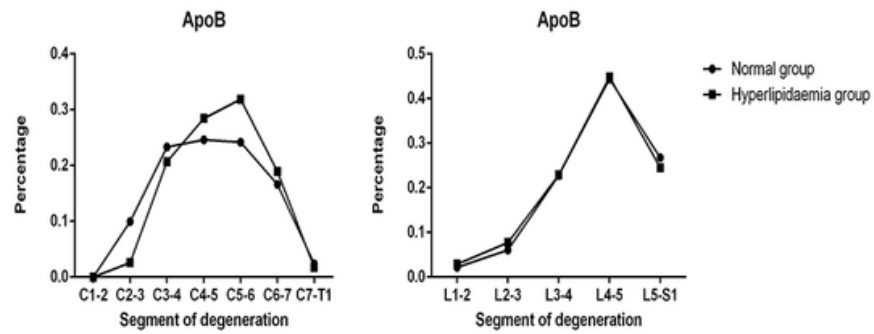

$\mathrm{F}$
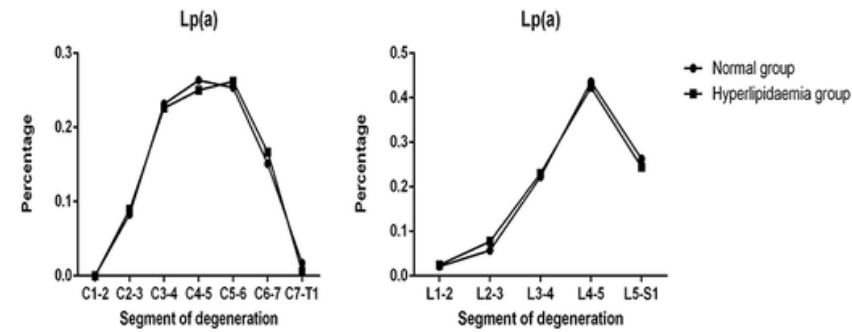

Figure 5

Hyperlipidaemia did not affect the incidence of intervertebral disc degenerated segment in cervical and lumbar spine The percentage of degenerated segment in spine with High TC (A); TG (B); LDL-C (C); Apo B (D); Apo Al (E); Apolipoprotein (F) group. 
A
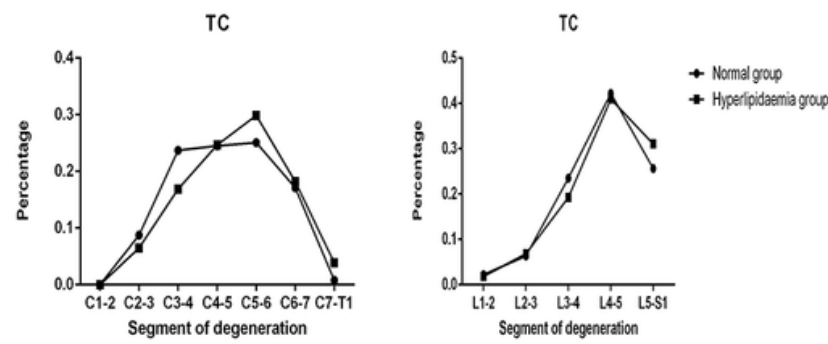

$\mathrm{C}$

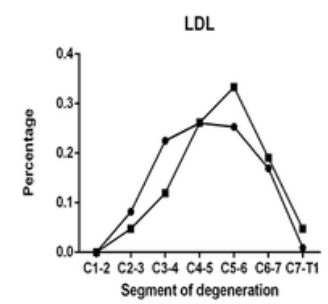

$\mathrm{E}$

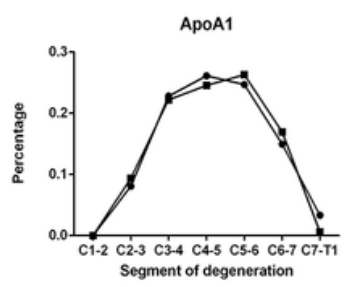

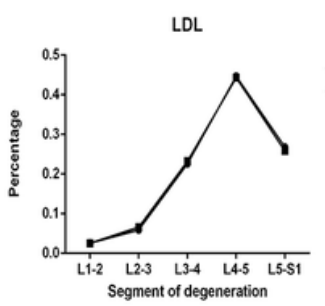

$\rightarrow$ Normal group

* Nyperlipidaemia group

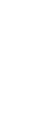

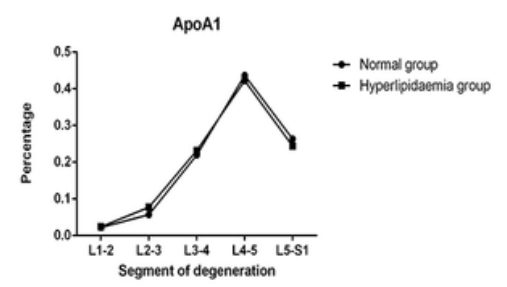

$\mathrm{B}$
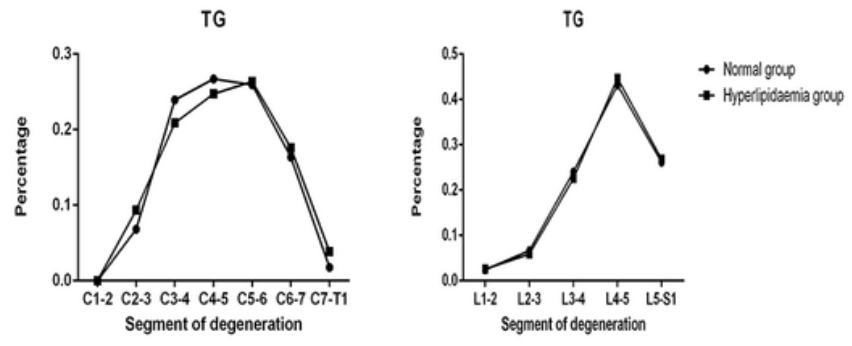

$\mathrm{D}$
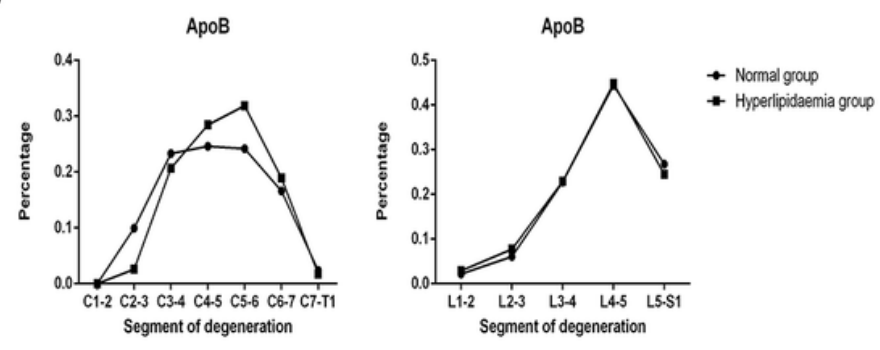

$\mathrm{F}$
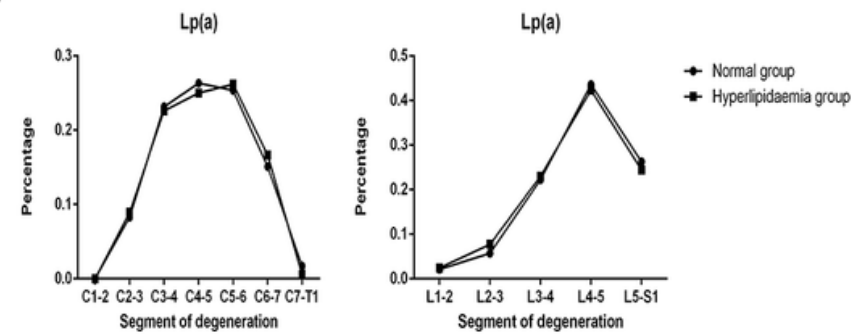

Figure 5

Hyperlipidaemia did not affect the incidence of intervertebral disc degenerated segment in cervical and lumbar spine The percentage of degenerated segment in spine with High TC (A); TG (B); LDL-C (C); Apo B (D); Apo Al (E); Apolipoprotein (F) group.

\section{Supplementary Files}

This is a list of supplementary files associated with this preprint. Click to download.

- Table.doc

- Table.doc 